Christian Patry

Stylianos E. Orfanos

Neysan Rafat

\section{Translational research in ARDS patients: new biological phenotypes}

Received: 22 July 2015

Accepted: 24 July 2015

Published online: 30 July 2015

(C) Springer-Verlag Berlin Heidelberg and ESICM 2015

C. Patry $\cdot$ N. Rafat $(\bowtie)$

Department of Pediatrics I, University Children's Hospital, University of Heidelberg, Im Neuenheimer Feld 430, 69120

Heidelberg, Germany

e-mail: Neysan.Rafat@med.uni-heidelberg.de

Tel.: +49 (6221) 56-36972

\section{S. E. Orfanos}

Second Department of Critical Care, Attikon University Hospital, University of Athens Medical School, 1, Rimini St., 12462 Haidari, Athens, Greece

\section{Clinical ARDS: Berlin definition and its "limitations"}

The acute respiratory distress syndrome (ARDS) represents a critical pulmonary disease entity which is pathophysiologically characterized by pulmonary endothelial injury and alveolar epithelial barrier disruption. In clinical practice intensive care givers use the Berlin definition of ARDS, established in 2012 in order to classify patients into three categories of severity on the basis of the $\mathrm{PaO}_{2} / \mathrm{FiO}_{2}$ ratio and other ventilatory parameters [1]. Depending on the degree of disease severity (from "mild" to "severe") several supportive therapeutic interventions are considered, ranging from protective ventilation to extracorporeal membrane oxygenation (ECMO) [2]. Currently, the Berlin definition does not directly register the underlying pathophysiological mechanisms in ARDS like endothelial barrier dysfunction or altered endothelial cell behavior in the course of the disease $[1,3]$. Regarding the development of pathophysiologically driven therapy options an integration of biomarkers for ARDS (like circulating endothelial cells, CECs) into the current clinical classification systems might be necessary.

\section{Translational research in ARDS}

With respect to endothelial and alveolar epithelial injury, translational research in ARDS mainly focusses on the interaction between neutrophils, platelets, macrophages, endothelial and alveolar epithelial cells [4]. Based on the vascular/inflammatory basis of ARDS pathophysiology, several treatment options like glucocorticoids, inhaled antioxidants, or protease inhibitors have been tested but lack proven overall effectiveness [5]. Promising new approaches towards the development of pathophysiologically driven therapies are based on cellular therapies such as bone marrow-derived progenitor cells $[6,7]$. Considering the importance of endothelial barrier dysfunction in ARDS, current research effort is being put into unveiling new vascular/endothelium-based targeted therapy options in ARDS [8].

\section{Pulmonary endothelium in ARDS}

To some extent constitutively but also partly upon exposure to activating stimuli the pulmonary endothelium regulates platelet aggregation, hemofluidity, and the vascular tone, it functions as a physiological barrier, participates in immune reactions, and exerts redox activities $[8,9]$.

Endothelial cell activation by specific stimuli is a central mechanism in the pathogenesis of ARDS [4]. There are several stimuli that may activate pulmonary endothelial cells (EC) such as mechanical stress or 
inflammatory cytokines like TNF- $\alpha$ and IL-1. ECs react upon activation with the expression of adhesion molecules (E- and P-selectins as well as ICAM-1 and PECAM1) in the context of neutrophil recruitment, with the increase of permeability, the promotion of platelet aggregation [8], and with the production of reactive oxygen species (ROS) [10]. In that way a pathophysiologically appropriate degree of EC activation in the course of ARDS may also lead to endothelial cell damage (giving rise to an increase of CEC levels), a situation which is aggravated by the activation of transmigrating neutrophils [8]. Endothelial cell damage is one reason for the increase in endothelial permeability in ARDS leading to alveolar fluid accumulation. This is one reason why current research efforts target on CECs as makers for endothelial damage in the course of EC activation in sepsis and ARDS [3]. Latest perceptions suggest that the serum level of CECs might prove to be a potent surrogate parameter for the severity of ARDS and the prognosis of the suffering patient [3].

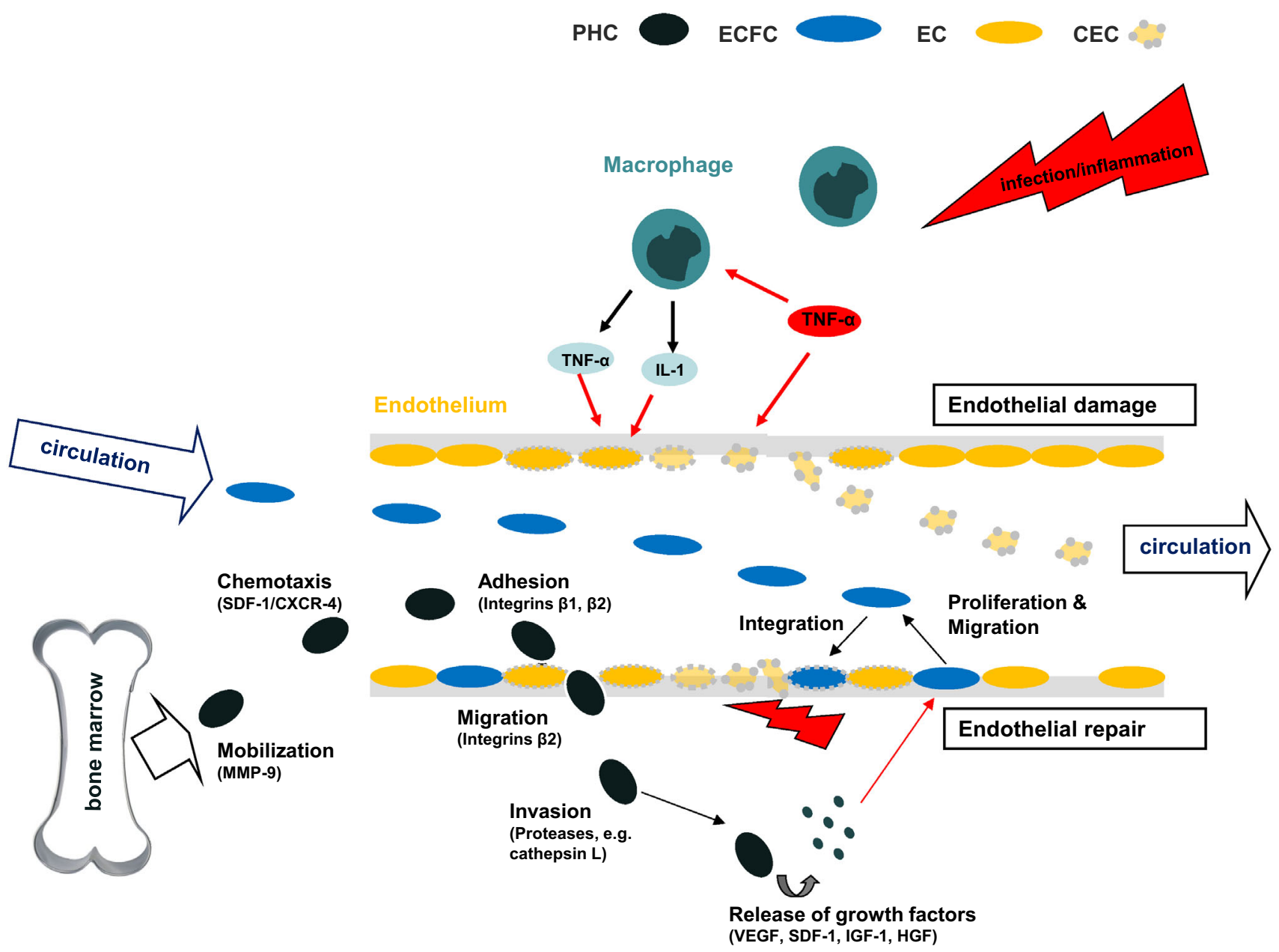

Fig. 1 Schematic illustration demonstrating the basic mechanisms of endothelial injury and repair. Endothelial injury can be induced by infection, encompassing many of the major inflammatory interactions among cytokines, macrophages, neutrophils (not shown here), and endothelium. Endothelial repair can be facilitated by subsets of endothelial progenitor cells. Recruitment and incorporation of proangiogenic hematopoietic cells (PHC) from bone marrow into ischemic or injured tissue requires a coordinated multistep process including mobilization, chemotaxis, adhesion to the endothelium, transendothelial migration, invasion, and in situ differentiation. PHC release growth factors which stimulate angiogenic activity of resting mature endothelial cells (EC). Different factors which are described to regulate the distinct steps are indicated. Endothelial colony forming cells (ECFC) have proliferative potential and divide and migrate to repair the injured area. $C E C$ circulating endothelial cells, $C X C R-4$ CXC-motif chemokine receptor-4, $E C$ endothelial cells, $E C F C$ endothelial colony forming cells, $H G F$ hepatocyte growth factor, $I G F-1$ insulin-like growth factor-1, $I L-1$ interleukin-1, $M M P-9$ matrix metallopeptidase-9, $P H C$ proangiogenic hematopoietic cells, LPS lipopolysaccharide, $S D F-1$ stromal derived factor-1, TNF- $\alpha$ tumor necrosis factor- $\alpha$, $V E G F$ vascular endothelial growth factor. Red arrow action, black arrow synthesis 


\section{Biomarkers for endothelial injury and their clinical significance}

Endothelial dysfunction in ARDS leads to shifts in activity or serum levels of endothelial-specific proteins, including angiotensin converting enzyme (ACE) [11], tissue factor pathway inhibitor [12], and von Willebrand factor [13]. Also, levels of endothelial progenitor cells are significantly increased in ARDS and show a correlation with improved survival [14]. In the study by Moussa et al. [3] the counts of CECs were significantly higher in ARDS patients compared to other critically ill patients. Within the ARDS group, patients with moderate and severe ARDS had significantly higher CEC counts on day 1 than patients with milder disease; therefore, the authors suggest CEC to be a useful marker of ARDS severity [3]. It remains to be determined whether CECs are useful as a biomarker of clinical outcome and/or treatment response in ARDS patients.

\section{Endothelial regeneration}

Simultaneously with endothelial injury, reconstitution of the endothelial layer is initiated. This reconstitution generally involves two biologic paradigms referred to as angiogenesis and vasculogenesis. Angiogenesis is the formation of new blood vessels via migration and proliferation of the endothelium of pre-existing vessels. The capacity of mature endothelial cells to proliferate, however, depends on the presence of endothelial colony forming cells (ECFC) that give rise to endothelial progeny [15]. Since there might be heterogeneity in the level of proliferative potential depending, e.g., on the vascular bed, adequate vascular repair might require additional support. In this context, adult vasculogenesis-the de novo formation of blood vessels from endothelial progenitor cells (EPC) - has been demonstrated to play a role (Fig. 1) [15]. Both cell types seem to be involved in the repair of endothelial denudation injury. While proangiogenic hematopoietic cells (PHC) are first recruited to the site of injury to facilitate repair, ECFC have vascularforming ability and integrate into the denuded vasculatures (Fig. 1) [15].

The critical question put forward in all studies involves the role that circulating and resident progenitor cells play in the resolution of injured pulmonary endothelium and increased pulmonary vascular permeability in the course of ARDS. It has been shown that the administration of bone marrow-derived progenitor cells (BMDPCs) decreases both the systemic and local inflammatory responses induced by endotoxin, and is associated with improved clinical outcomes [6, 7]. Therefore, the differential release of progenitor cells from the bone marrow offers particular therapeutic potential. Strategies to manipulate the microenvironment to enhance lung repair, particularly in patients who are immunocompromised and have inadequate bone marrow responses, would provide obvious therapeutic benefits. The particular BMDPC populations that are critical in repairing lung injuries remain to be determined. This could lead to important approaches in cell-based treatments where effective BMDPCs are infused in patients with ARDS and other lung diseases.

\section{Compliance with ethical standards}

Conflicts of interest On behalf of all authors, the corresponding author states that there is no conflict of interest.

\section{References}

1. Ranieri VM, Rubenfeld GD, Thompson BT et al (2012) Acute respiratory distress syndrome: the Berlin definition. JAMA 307:2526-2533

2. Ferguson ND, Fan E, Camporota $L$ et al (2012) The Berlin definition of ARDS: an expanded rationale, justification, and supplementary material. Intensive Care Med 38:1573-1582

3. Moussa MD, Santonocito C, Fagnoul D et al (2015) Evaluation of endothelial damage in sepsis-related ARDS using circulating endothelial cells. Intensive Care Med 41:231-238

4. Matthay MA, Zemans RL (2011) The acute respiratory distress syndrome: pathogenesis and treatment. Annu Rev Pathol 6:147-163
5. Cepkova M, Matthay MA (2006) Pharmacotherapy of acute lung injury and the acute respiratory distress syndrome. J Intensive Care Med 21:119-143

6. Rafat N, Dacho C, Kowanetz G et al (2014) Bone marrow-derived progenitor cells attenuate inflammation in lipopolysaccharide-induced acute respiratory distress syndrome. BMC Res Notes 7:613

7. Rafat N, Kowanetz G, Krebs J et al (2014) Therapeutic effects of bone marrow-derived progenitor cells in lipopolysaccharide-induced acute respiratory distress syndrome. J Pulm Respir Med 4:2
8. Orfanos SE, Mavrommati I, Korovesi I, Roussos C (2004) Pulmonary endothelium in acute lung injury: from basic science to the critically ill. Intensive Care Med 30:1702-1714

9. Maniatis NA, Kotanidou A, Catravas JD, Orfanos SE (2008) Endothelial pathomechanisms in acute lung injury. Vascul Pharmacol 49:119-133

10. Bhatia M, Moochhala S (2004) Role of inflammatory mediators in the pathophysiology of acute respiratory distress syndrome. J Pathol 202:145-156 
11. Orfanos SE, Armaganidis A, Glynos C et al (2000) Pulmonary capillary endothelium-bound angiotensinconverting enzyme activity in acute lung injury. Circulation 102:2011-2018

12. Sabharwal AK, Bajaj SP, Ameri A et al (1995) Tissue factor pathway inhibitor and von Willebrand factor antigen levels in adult respiratory distress syndrome and in a primate model of sepsis. Am J Respir Crit Care Med 151:758-767
13. Ware LB, Eisner MD, Thompson BT et al (2004) Significance of von Willebrand factor in septic and nonseptic patients with acute lung injury. Am J Respir Crit Care Med 170:766-772

14. Burnham EL, Taylor WR, Quyyumi AA et al (2005) Increased circulating endothelial progenitor cells are associated with survival in acute lung injury. Am J Respir Crit Care Med 172:854-860
15. Rafat N, Tönshoff B, Bierhaus A, Beck GC (2013) Endothelial progenitor cells in regeneration after acute lung injury: do they play a role? Am J Respir Cell Mol Biol 48:399-405 